\title{
Integrated analysis of differential gene expression profiles in hippocampi to identify candidate genes involved in Alzheimer's disease
}

\author{
WANHUA HU ${ }^{1 *}$, XIAODONG LIN $^{2 *}$ and KELONG CHEN ${ }^{1}$ \\ ${ }^{1}$ Department of Neurology, Wenzhou Hospital of Traditional Chinese Medicine, Wenzhou, Zhejiang 325000; \\ ${ }^{2}$ Department of Traditional Chinese Internal Medicine, Wenzhou Seventh People's Hospital, \\ Wenzhou, Zhejiang 325005, P.R. China
}

Received October 21, 2014; Accepted July 28, 2015

DOI: $10.3892 / \mathrm{mmr} .2015 .4271$

\begin{abstract}
Alzheimer's disease (AD) is a complex neurodegenerative disorder with largely unknown genetic mechanisms. Identifying altered neuronal gene expression in AD may provide diagnostic or therapeutic targets for AD. The present study aimed to identify differentially expressed genes (DEGs) and their further association with other biological processes that regulate causative factors for AD. The present study performed an integrated analysis of publicly available gene expression omnibus datasets of AD hippocampi. Gene ontology (GO) enrichment analyses, Kyoto Encyclopedia of Genes and Genomes (KEGG) pathway analysis and Protein-Protein interaction (PPI) network analysis were performed. The present study detected 295 DEGs (109 upregulated and 186 downregulated genes) in hippocampi between AD and control samples by integrating four datasets of gene expression profiles of hippocampi of patients with AD. Respiratory electron transport chain (GO: 0022904; $\left.\mathrm{P}=1.64 \times 10^{-11}\right)$ was the most significantly enriched GO term among biological processes, while for molecular functions, the most significantly enriched GO term was that of protein binding (GO: 0005515; $\mathrm{P}=3.03 \times 10^{-29}$ ), and for cellular components, the most significantly enriched GO term was that of the cytoplasm (GO:
\end{abstract}

Correspondence to: Dr Wanhua $\mathrm{Hu}$, Department of Neurology, Wenzhou Hospital of Traditional Chinese Medicine, 9 Liuhongqiaojiaowei Road, Lucheng, Wenzhou, Zhejiang 325000, P.R. China

E-mail: wanhua_hu@163.com

Dr Xiaodong Lin, Department of Traditional Chinese Internal Medicine, Wenzhou Seventh People's Hospital, 552 East Xishan Road, Wenzhou, Zhejiang 325005, P.R. China

E-mail: lin_xiaodong166@126.com

*Contributed equally

Key words: integrated analysis, expression data, Alzheimer's disease, hippocampus, microarray, differentially expressed genes
0005737; $\left.\mathrm{P}=8.67 \times 10^{-33}\right)$. The most significant pathway in the KEGG analysis was oxidative phosphorylation $\left(\mathrm{P}=1.61 \times 10^{-13}\right)$. PPI network analysis showed that the significant hub proteins contained $\beta$-actin (degree, 268), hepatoma-derived growth factor (degree, 218) and WD repeat-containing protein 82 (degree, 87). The integrated analysis performed in the present study serves as a basis for identifying novel drug targets to develop improved therapies and interventions for common and devastating neurological diseases such as AD.

\section{Introduction}

Alzheimer's disease (AD) is one of the most common and complex neurodegenerative disorders and is characterized by a progressive decline of memory and cognition (1). The disease is defined by specific neuropathological changes of neurofibrillary tangles (NFT) and amyloid plaques that accumulate in vulnerable brain regions $(2,3)$. Neurodegeneration in the development of $\mathrm{AD}$ varies substantially across cell types and regions. Of note, it has been demonstrated that hippocampal CA1 pyramidal neurons are particularly vulnerable to neurodegeneration and bear NFTs during the early stages of $\mathrm{AD}(4,5)$; however, the underlying mechanisms of their degeneration have remained elusive.

$\mathrm{AD}$ is thought to be caused by the dysregulation of a large number of genes and the consequent alteration of their complex interactions, which finally contributes to the broad spectrum of disease phenotypes (6-9). Microarray technology, which provides researchers with a tool to assess the expression levels of thousands of genes simultaneously, offers the possibility of gaining insight into gene networks disturbed in intricate human disease such as $\mathrm{AD}$, and to obtain possible molecular clues regarding the underlying mechanisms of the pathophysiology of AD. Previous studies have used this technique to more comprehensively enhance the knowledge of the cellular and molecular changes underlying AD (10-14). Although these studies have yielded significant novel insights, inconsistencies are present across these studies due to limitations based on small sample sizes and various results obtained by different groups with different laboratory protocols, microarray platforms and microarray data interpretations (15). In 
Table I. Characteristics of the individual studies.

\begin{tabular}{|c|c|c|c|c|c|}
\hline GEO ID & Platform & $\begin{array}{c}\text { Samples (n) } \\
\text { (cases:controls) }\end{array}$ & Country & Year & Author \\
\hline GSE29378 & $\begin{array}{l}\text { GPL6947 Illumina HumanHT-12 } \\
\text { V3.0 expression beadchip }\end{array}$ & $31: 32$ & USA & 2013 & Miller JA (22) \\
\hline GSE36980 & $\begin{array}{l}\text { GPL6244 [HuGene-1_0-st] } \\
\text { Affymetrix Human Gene 1.0 ST Array }\end{array}$ & $7: 10$ & Japan & 2013 & Hokama M (23) \\
\hline GSE5281 & $\begin{array}{l}\text { GPL570 [HG-U133_Plus_2] Affymetrix } \\
\text { Human Genome U133 Plus 2.0 Array }\end{array}$ & $13: 10$ & USA & 2007 & Liang WS (24) \\
\hline GSE1297 & $\begin{array}{l}\text { GPL96 [HG-U133A] Affymetrix } \\
\text { Human Genome U133A Array }\end{array}$ & 22:9 & USA & 2004 & Blalock EM (12) \\
\hline
\end{tabular}

GEO, Gene Expression Omnibus.

view of this, the present study integrated hippocampus gene expression datasets from multiple AD microarray studies to overcome these limitations of individual studies, resolve inconsistencies and provide significant novel insight into the complex biological processes involved in AD.

\section{Materials and methods}

Identification of eligible gene expression profiles of hippocampi of patients with AD. Hippocampal gene expression profiling studies in patients with $\mathrm{AD}$ were identified by searching the Gene Expression Omnibus database (GEO; http://www.ncbi.nlm.nih.gov/geo) (16). The following key words and their combinations were used: 'Alzheimer's disease', 'hippocampus', 'gene expression' and 'microarray.' Only experimental studies that had performed hippocampal gene expression profiling in patients with $\mathrm{AD}$ as well as normal control (NC) subjects were used. Non-human studies, review articles and integrated analyses of expression profiles were excluded.

Data preprocessing. Normalization is crucial for comparing different microarray datasets. The heterogeneity caused by different microarray platforms, gene nomenclature and clinical samples may make it difficult to compare the expression data directly. However, inappropriate normalization may contribute to the skewing of results and reduce their statistical significance. Consequently, a global normalization approach to minimize any inconsistencies should be included. For this propose, MATLAB Bioinformatics Toolbox was used in the present study to pre-process the raw microarray data of each study by Quantile normalization and $\log 2$ transformation to obtain intensity values.

Statistical analysis. MATLAB software, version 2013a (MathWorks, Natick, MA) was used to identify the differently expressed probe sets in the hippocampal tissues of patients with AD compared to those of NC subjects. A gene-specific $\mathrm{t}$-test was performed, followed by calculation of the P-value and the effect size of the individual microarray study. Fisher's combined probability method was used to combine P-values from multiple studies, and the random effects model was used to combine effect sizes from multiple studies. Genes with an effect size $>0.8$ and a P-value $<0.01$ were selected as the significantly differentially expressed genes (DEGs).

Functional annotation of DEGs. To gain insight into the biological functions of DEGs, gene ontology (GO) classification was performed. GO provides a common descriptive framework as well as functional annotation and classification for analyzing the gene expression datasets. Furthermore, Kyoto Encyclopedia of Genes and Genomes (KEGG; http://www. genome.jp/kegg/) pathway enrichment analysis was performed to map the potential pathways of the DEGs. The KEGG pathway database is a recognized and comprehensive database, which includes an extensive variety of biochemical pathways (17). The online-based software GENECODIS, version 3 was utilized in the present analysis (18).

Protein-protein interactions (PPIs) network construction. PPI analysis allows for the assessment of protein functions at the molecular level, which are divided into the categories of cellular growth, development, metabolism, differentiation and apoptosis (19). The detection of key protein-interacting ions in the PPI networks of AD is important for the interpretation of cellular regulatory mechanisms in the development of the disease (20). The present study adopted the Search Tool for the Retrieval of Interacting Genes/Proteins (http://www.string-db. org/), a database of known and predicted protein interactions, to construct the PPI network and then visualized the distribution characteristics of the top 10 up- and downregulated DEGs in the network with Cytoscape software, version 3.2.0 (21).

\section{Results}

Identification of DEGs in hippocampi of patients with $A D$. The present study collected a total of four datasets of gene expression profiles in hippocampi of patients with $\mathrm{AD}$ according to the inclusion criteria; in total, data on the gene expression in 73 samples from patients with AD and 61 samples from control subjects were analyzed. The studies containing the individual hippocampal expression profiles in patients with AD are listed in Table I (12,22-24). A total of 11,494 genes from four expression profiling studies were 
Table II. Top 10 most significantly up- or down-regulated differentially expressed genes.

\begin{tabular}{|c|c|c|c|c|}
\hline Gene ID & Gene symbol & Official full name & P-value & Effect size \\
\hline \multicolumn{5}{|c|}{ Upregulated genes } \\
\hline 51663 & ZFR & Zinc finger RNA binding protein & $1.12 \times 10^{-6}$ & 0.94853 \\
\hline 2669 & GEM & $\begin{array}{l}\text { GTP binding protein overexpressed } \\
\text { in skeletal muscle }\end{array}$ & $1.21 \times 10^{-6}$ & 1.1503 \\
\hline 6277 & S100A6 & S100 calcium binding protein A6 & $2.74 \times 10^{-6}$ & 1.0918 \\
\hline 80335 & WDR82 & WD repeat domain 82 & $3.05 \times 10^{-6}$ & 0.80325 \\
\hline 5209 & PFKFB3 & $\begin{array}{l}\text { 6-Phosphofructo-2-kinase/fructose-2, } \\
\text { 6-Biphosphatase } 3\end{array}$ & $3.24 \times 10^{-6}$ & 1.1908 \\
\hline 3895 & KTN1 & Kinectin 1 (kinesin receptor) & $3.36 \times 10^{-6}$ & 1.1643 \\
\hline 3068 & HDGF & $\begin{array}{l}\text { Hepatoma-derived growth factor } \\
\text { (high-mobility group protein 1-like) }\end{array}$ & $4.57 \times 10^{-6}$ & 1.1580 \\
\hline 2077 & ERF & Ets2 repressor factor & $5.16 \times 10^{-6}$ & 1.0677 \\
\hline 7049 & TGFBR3 & Transforming growth factor, beta receptor III & $5.34 \times 10^{-6}$ & 1.1818 \\
\hline 5042 & PABPC3 & Poly(A) binding protein, cytoplasmic 3 & $7.09 \times 10^{-6}$ & 1.0283 \\
\hline \multicolumn{5}{|c|}{ Downregulated genes } \\
\hline 22820 & COPG1 & Coatomer protein complex, subunit gamma & $8.69 \times 10^{-8}$ & 1.3017 \\
\hline 58189 & WFDC1 & WAP four-disulfide core domain 1 & $4.19 \times 10^{-7}$ & 1.3844 \\
\hline 10093 & ARPC4 & $\begin{array}{l}\text { Tubulin tyrosine ligase-like family, member } 3 \text {; } \\
\text { actin related protein } 2 / 3 \text { complex, } \\
\text { subunit } 4,20 \mathrm{kDa}\end{array}$ & $7.43 \times 10^{-7}$ & 1.1458 \\
\hline 60 & ACTB & Actin, beta & $1.08 \times 10^{-6}$ & 1.1657 \\
\hline 9158 & FIBP & $\begin{array}{l}\text { fibroblast growth factor (acidic) } \\
\text { intracellular binding protein }\end{array}$ & $1.15 \times 10^{-6}$ & 1.1794 \\
\hline 56993 & TOMM22 & $\begin{array}{l}\text { Translocase of outer mitochondrial } \\
\text { membrane } 22 \text { homolog (yeast) }\end{array}$ & $2.35 \times 10^{-6}$ & 1.0773 \\
\hline 2537 & IFI6 & Interferon, alpha-inducible protein 6 & $2.57 \times 10^{-6}$ & 1.1754 \\
\hline 9556 & C14orf2 & Chromosome 14 open reading frame 2 & $2.72 \times 10^{-6}$ & 1.1734 \\
\hline 55837 & EAPP & E2F-associated phosphoprotein & $3.11 \times 10^{-6}$ & 1.0270 \\
\hline 5889 & RAD51C & RAD51 homolog C (S. cervisiae $)$ & $5.16 \times 10^{-6}$ & 1.0461 \\
\hline
\end{tabular}

assessed. For the purpose of global normalization, the raw microarray data were pre-processed by Quantile normalization and $\log 2$ transformation to obtain intensity values for each probe, which were used in the gene expression profiling. Subsequently, MATLAB software was utilized to identify DEGs in hippocampi between patients with AD and control subjects. Finally, a total of 295 DEGs were regarded as significantly differentially expressed between samples of patients with AD and NC subjects (109 upregulated and 186 downregulated genes) when the threshold was set as $\mathrm{P}<0.01$ and effect size $>0.8$. A list of the top 10 most significantly upor downregulated genes is presented in Table II. The pattern of expressional changes of the top 50 most significantly DEGs is displayed in a heat map in Fig. 1.

The upregulated gene with the lowest P-value was ZFR, which is mainly expressed in neural tissue, but also weakly expressed in other tissue types $(25,26)$, suggesting a neuronal function. A recent study identified ZFR as a putative genes associated with hereditary spastic paraplegias by using whole-exome sequencing (27). The downregulated gene with the lowest P-value was COPG1, whose function has yet to be determined.
Functional annotation. To investigate the biological roles of the DEGs in the hippocampi of patients with AD, the present study performed a categorized GO enrichment analysis. GO provides a common descriptive framework and functional annotation of the gene datasets. GO categories are separated into three groups: Biological processes, cellular components and molecular function. The present study examined GO categories separately using the web-based software GENECODIS. The results showed that genes associated with the respiratory electron transport chain (GO: 0022904; $\mathrm{P}=1.64 \times 10^{-11}$ ) and gluconeogenesis (GO: 0006094; $\left.\mathrm{P}=2.84 \times 10^{-5}\right)$ were significantly enriched among biological processes, while for molecular functions, protein binding (GO:0005515; $\mathrm{P}=3.03 \times 10^{-29}$ ) and nucleotide binding (GO: 0000166; $\mathrm{P}=5.41 \times 10^{-14}$ ) were significantly enriched, and with regard to cellular components, genes associated with the cytoplasm (GO:0005737; $\mathrm{P}=8.67 \times 10^{-33}$ ) and mitochondrion (GO: 0005739; $\mathrm{P}=1.00 \times 10^{-23}$ ) were significantly enriched (Table III, Fig. 2A).

The present study subsequently performed a KEGG pathway enrichment analysis in order to further evaluate the biological roles of the DEGs. A hypergeometric test 


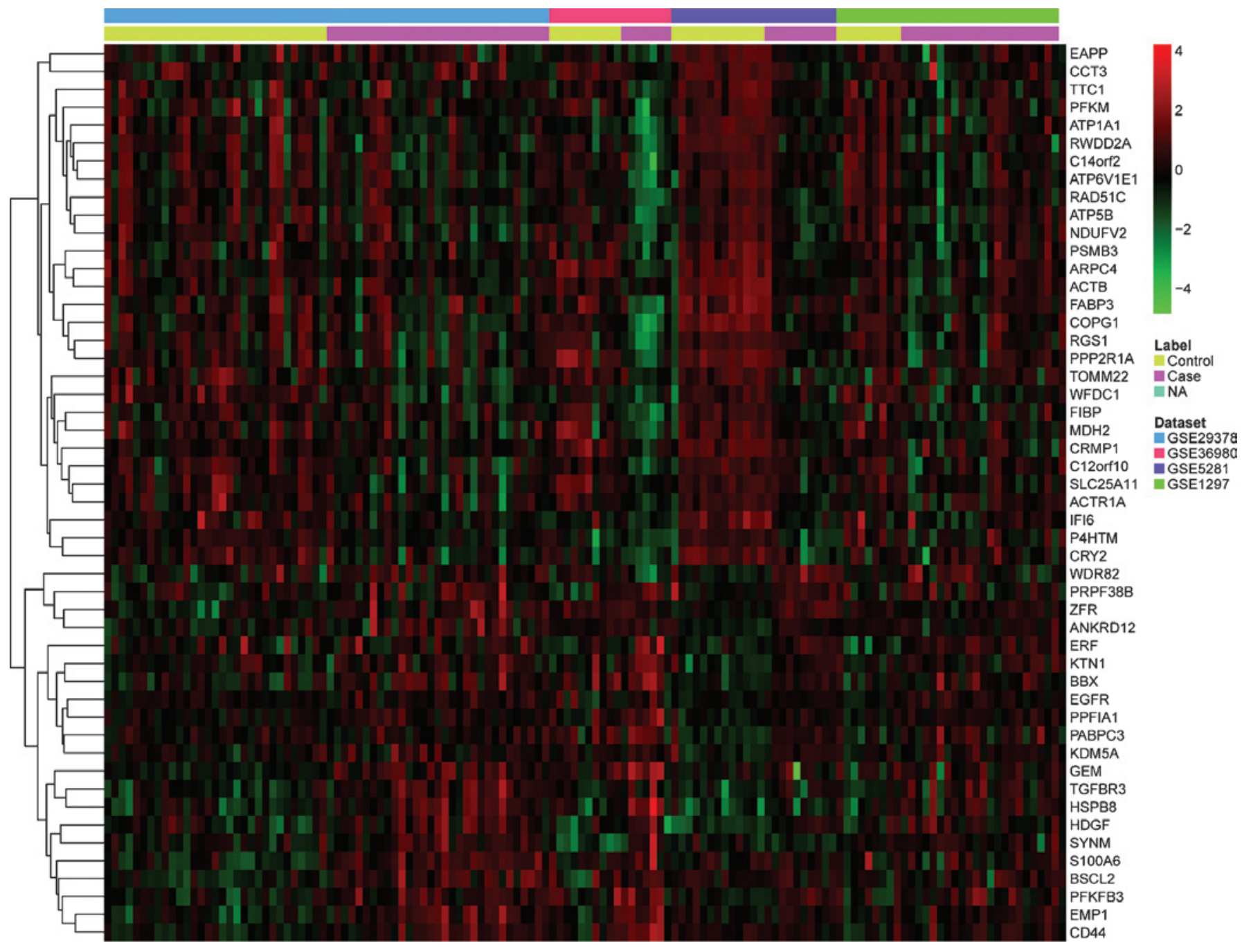

Figure 1. Heat map visualization of the patterns of expressional changes for the top 50 most significantly differentially expressed genes across various datasets.

A

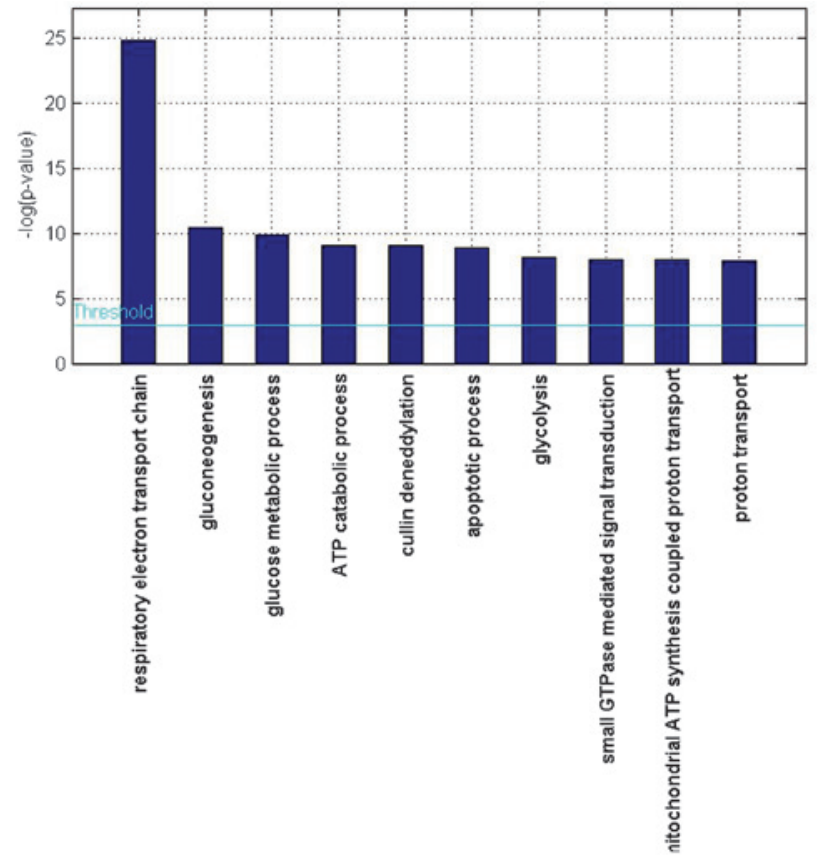

B

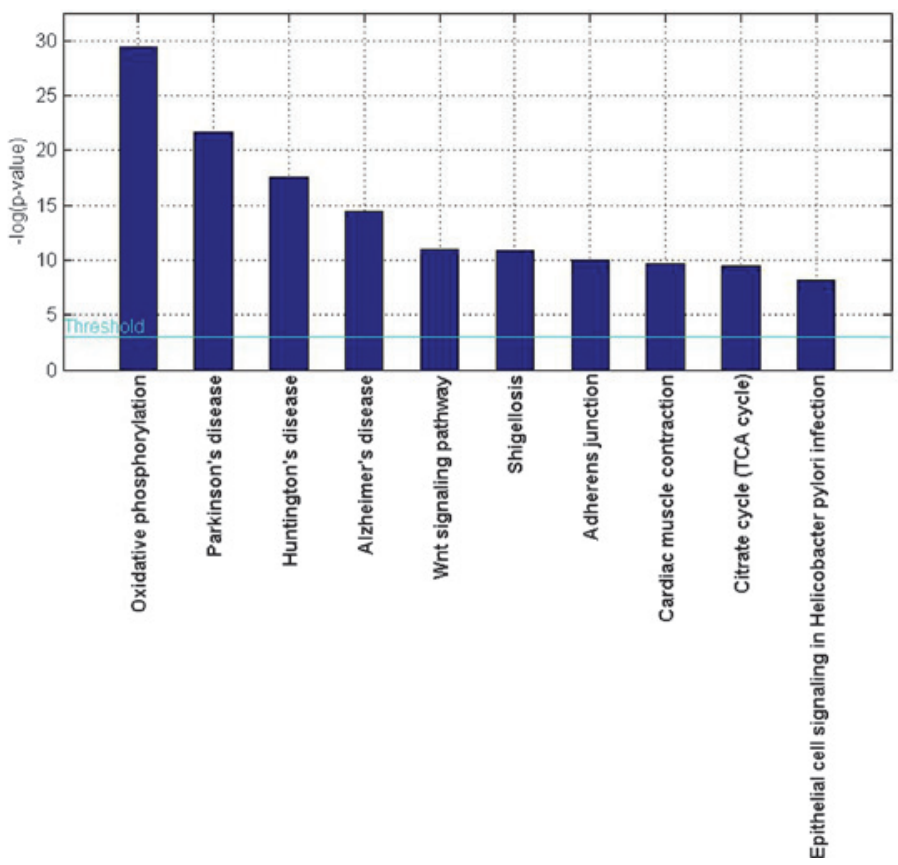

Figure 2. Functional annotation of significantly enriched differentially expressed genes. (A) The top 10 enriched gene ontology categories for biological processes; (B) The top 10 enriched Kyoto Encyclopedia of Genes and Genomes pathways. 
Table III. GO terms of differentially expressed genes (top 15).

\begin{tabular}{|c|c|c|c|}
\hline GO ID & GO term & No. of genes & FDR \\
\hline \multicolumn{4}{|c|}{ Biological processes } \\
\hline GO:0022904 & Respiratory electron transport chain & 14 & $1.64 \times 10^{-11}$ \\
\hline GO:0006094 & Gluconeogenesis & 7 & $2.84 \times 10^{-5}$ \\
\hline GO:0006006 & Glucose metabolic process & 9 & $4.94 \times 10^{-5}$ \\
\hline GO:0006200 & ATP catabolic process & 8 & $1.15 \times 10^{-4}$ \\
\hline GO:0010388 & Cullin deneddylation & 4 & $1.18 \times 10^{-4}$ \\
\hline GO:0006915 & Apoptotic process & 19 & $1.35 \times 10^{-4}$ \\
\hline GO:0006096 & Glycolysis & 6 & $2.91 \times 10^{-4}$ \\
\hline GO:0007264 & Small GTPase mediated signal transduction & 13 & $3.44 \times 10^{-4}$ \\
\hline GO:0042776 & Mitochondrial ATP synthesis coupled proton transport & 4 & $3.61 \times 10^{-4}$ \\
\hline GO:0015992 & Proton transport & 6 & $3.84 \times 10^{-4}$ \\
\hline GO:0007165 & Signal transduction & 26 & $7.19 \times 10^{-4}$ \\
\hline GO:0006120 & Mitochondrial electron transport, NADH to ubiquinone & 5 & $1.01 \times 10^{-3}$ \\
\hline GO:0006810 & Transport & 17 & $1.26 \times 10^{-3}$ \\
\hline GO:0048146 & Positive regulation of fibroblast proliferation & 5 & $1.30 \times 10^{-3}$ \\
\hline GO:0016071 & mRNA metabolic process & 10 & $1.40 \times 10^{-3}$ \\
\hline \multicolumn{4}{|c|}{ Molecular function } \\
\hline GO:0005515 & Protein binding & 117 & $3.03 \times 10^{-29}$ \\
\hline GO:0000166 & Nucleotide binding & 59 & $5.41 \times 10^{-14}$ \\
\hline GO:0005524 & ATP binding & 37 & $5.48 \times 10^{-7}$ \\
\hline GO:0005525 & GTP binding & 16 & $1.32 \times 10^{-5}$ \\
\hline GO:0046961 & Proton-transporting ATPase activity, rotational mechanism & 5 & $4.49 \times 10^{-5}$ \\
\hline GO:0003924 & GTPase activity & 12 & $4.55 \times 10^{-5}$ \\
\hline GO:0015631 & Tubulin binding & 5 & $8.56 \times 10^{-5}$ \\
\hline GO:0046933 & $\begin{array}{l}\text { Hydrogen ion transporting ATP synthase activity, } \\
\text { rotational mechanism }\end{array}$ & 4 & $3.08 \times 10^{-4}$ \\
\hline GO:0005509 & Calcium ion binding & 18 & $5.87 \times 10^{-4}$ \\
\hline GO:0008137 & NADH dehydrogenase (ubiquinone) activity & 5 & $6.24 \times 10^{-4}$ \\
\hline GO:0003713 & Transcription coactivator activity & 10 & $6.81 \times 10^{-4}$ \\
\hline GO:0022857 & Transmembrane transporter activity & 5 & $8.83 \times 10^{-4}$ \\
\hline GO:0005516 & Calmodulin binding & 8 & $1.17 \times 10^{-3}$ \\
\hline GO:0047485 & Protein N-terminus binding & 6 & $1.81 \times 10^{-3}$ \\
\hline GO:0003878 & ATP citrate synthase activity & 2 & $1.87 \times 10^{-3}$ \\
\hline \multicolumn{4}{|c|}{ Cellular components } \\
\hline GO:0005737 & Cytoplasm & 133 & $8.67 \times 10^{-23}$ \\
\hline GO:0005739 & Mitochondrion & 60 & $1.00 \times 10^{-23}$ \\
\hline GO:0005829 & Cytosol & 62 & $6.03 \times 10^{-16}$ \\
\hline GO:0005743 & Mitochondrial inner membrane & 25 & $1.38 \times 10^{-15}$ \\
\hline GO:0005634 & Nucleus & 101 & $4.71 \times 10^{-14}$ \\
\hline GO:0005856 & Cytoskeleton & 26 & $7.29 \times 10^{-7}$ \\
\hline GO:0005625 & Soluble fraction & 17 & $1.64 \times 10^{-6}$ \\
\hline GO:0016020 & Membrane & 66 & $3.04 \times 10^{-6}$ \\
\hline GO:0005886 & Plasma membrane & 60 & $3.94 \times 10^{-6}$ \\
\hline GO:0005759 & Mitochondrial matrix & 12 & $4.18 \times 10^{-6}$ \\
\hline GO:0005654 & Nucleoplasm & 25 & $4.28 \times 10^{-6}$ \\
\hline GO:0045121 & Membrane raft & 9 & $3.28 \times 10^{-5}$ \\
\hline GO:0005730 & Nucleolus & 31 & $4.98 \times 10^{-5}$ \\
\hline GO:0005753 & Mitochondrial proton-transporting ATP synthase complex & 4 & $1.58 \times 10^{-4}$ \\
\hline GO:0030054 & Cell junction & 15 & $2.50 \times 10^{-4}$ \\
\hline
\end{tabular}

FDR, false discovery rate; GO, gene ontology. 


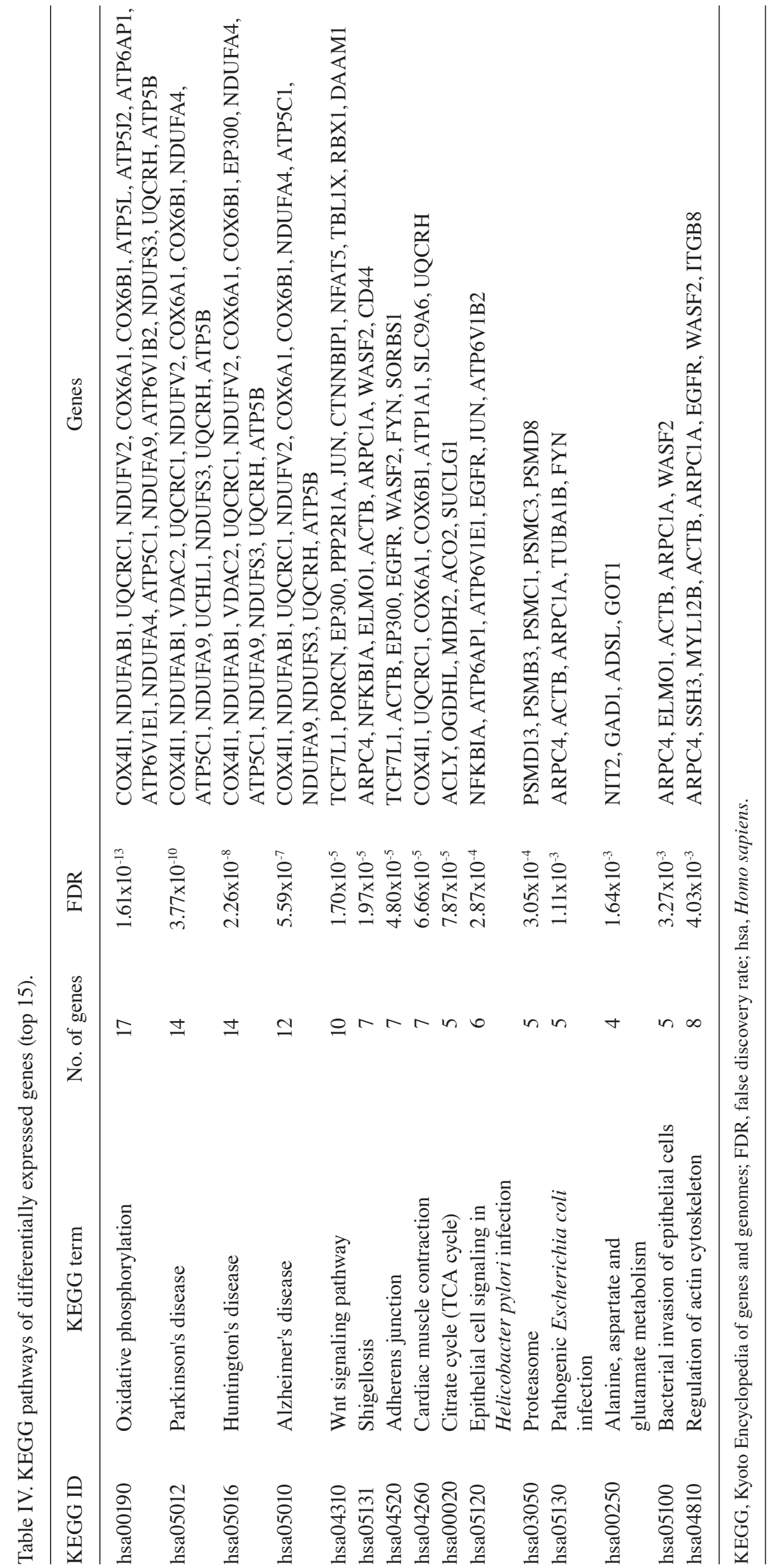




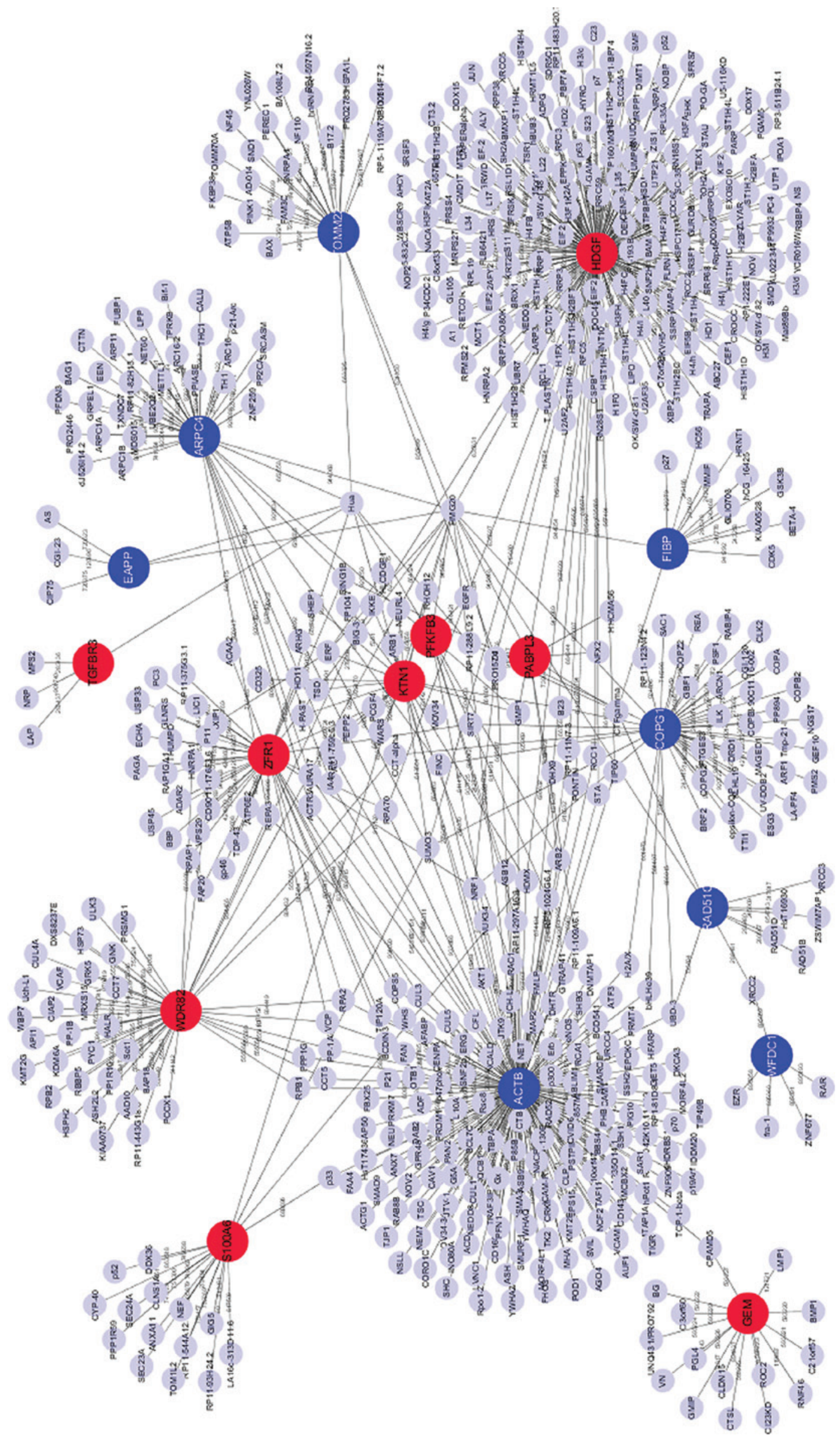

Figure 3. Constructed protein-protein interaction networks of the top 10 up- and down-regulated DEGs. Nodes represent proteins and edges represent interactions between two proteins. Red- and green-colored nodes represent products of up- and down-regulated DEGs, respectively. Blue nodes denote products of genes predicted to interact with the DEGs. DEG, differentially-expressed gene. 
with $\mathrm{P}<0.05$ was used as the criterion for pathway detection. According to the KEGG analysis, oxidative phosphorylation was the most significant pathway $\left(\mathrm{P}=1.61 \times 10^{-13}\right)$. Furthermore, pathways involved in Parkinson's disease $\left(\mathrm{P}=3.77 \times 10^{-10}\right)$ and Huntington's disease $\left(\mathrm{P}=2.26 \times 10^{-8}\right)$ were also highly enriched (Table IV, Fig. 2B).

PPI network construction. The present study established the PPI networks of the top 10 upregulated and downregulated DEGs using Cytoscape software. The interaction network included 863 nodes and 1,304 edges. In the PPI network, degrees of interaction were defined to determine the number of neighbors a node directly connected to, and nodes with a high degree of interaction were defined as hub proteins. The significant hub proteins included $\beta$-actin (ACTB; degree, 268), hepatoma-derived growth factor (HDGF; degree, 218) and WD repeat-containing protein 82 (WDR82; degree, 87) (Fig. 3).

\section{Discussion}

The present study aimed to identify altered hippocampus gene expression and their further association with other biological processes that regulate causative factors for $\mathrm{AD}$ to provide diagnostic factors or therapeutic targets of AD. An integrated analysis of DEGs from four publicly available GEO datasets of hippocampi from patients with $\mathrm{AD}$ was performed. In total, 295 genes were consistently differentially expressed across the studies with 109 upregulated genes and 186 downregulated genes. The upregulated gene with the lowest P-value was ZFR, which is mainly expressed in neural tissue $(25,26)$ and may therefore have a role in neuronal function. A recent study identified ZFR as a putative gene associated with hereditary spastic paraplegias by using whole-exome sequencing (27), and from this, the present study deduced that ZFR may be implicated in the underlying processes of $\mathrm{AD}$, which is required to be confirmed by further experiments. The downregulated gene with the lowest P-value was COPG1, whose function remains to be elucidated.

In line with previous studies, certain genes identified in the present study have been closely associated with the development of AD, including S100A6 and TGFBR3. A study on the roles of S100 family proteins in nervous system function and disease found that mRNA expression levels of six family members (S100A1, S100B, S100A6, S100A10, S100A4, S100A13) displayed a 100-fold range in mouse brains, five of which (S1100A1, S100A6, S100A10, S100A13, and S100B) showed age-dependent increases in adult mice that ranged from 5- to 20-fold (28). S100A6-protein immunoreactivity was found to be specifically located within astrocytes associated to amyloid plaques in an APP/London transgenic mouse model of AD, as well as in the brains of patients with AD. S100A6 was upregulated in the amygdala as well as in hippocampal regions (29). Another study detected that biglycan proteoglycans were upregulated in familial AD, while TGFBR3 was markedly downregulated in sporadic AD fibroblasts. Furthermore, the differential expression of TGFBR3 in familial AD and sporadic AD cells was associated with the severity of AD (30).

In the present study, the results of the PPI network analysis of the top 10 upregulated and downregulated DEGs indicated that the significant hub proteins included ACTB, HDGF and WDR82. ACTB, which encodes $\beta$-actin, is a candidate reference gene for normalization of target gene expression in polymerase chain reaction (PCR) analysis due to its high conservation. A previous study determined the mRNA levels of ACTB and other genes in the frontal cortex of patients with AD and control subjects using PCR analysis with SYBR Green technology to identify suitable endogenous reference genes in human post-mortem brain tissues for the expression analysis of potential candidate genes associated with AD (31); according to this study, ACTB was the least suitable candidate with reliable expression among a set of suitable endogenous reference genes due to low expression stability in the frontal cortex of AD (32). Of note, the actin cytoskeleton has been reported to have an important role in AD pathology by mediating synaptic degeneration (32).

In order to elucidate the biological roles of the DEGs in $\mathrm{AD}$, a categorized GO enrichment analysis was performed in the present study. The results showed that the respiratory electron transport chain was the most significantly enriched GO category for biological processes. To further evaluate the biological role for the DEGs, the present study performed a KEGG pathway enrichment analysis. According to the KEGG analysis, the most significantly enriched pathway was oxidative phosphorylation. A previous study provided evidence of neuronal metabolic impairments at the transcriptomic and protein level in the brains of patients with AD (33), which was ascribed to the downregulation of mitochondria-associated genes, in particular, oxidative phosphorylation genes in consistency with the fact that AD is a degenerative disease. Furthermore, the present study found that the pathways of several neurodegenerative diseases, including Parkinson's disease, Huntington's disease and Alzheimer's disease, were also highly enriched according to the KEGG pathway enrichment analysis, which was due to dysregulation of genes associated with mitochondrial energy metabolism, including COX4I1, NDUFAB1, UQCRC1, NDUFV2, COX6A1, COX6B1. This finding validated the integrated analysis methods used in the present study.

It is noteworthy that the present study had several limitations. The heterogeneity of the datasets used may have distorted the analysis, as clinical samples may have been heterogeneous with regard to clinical activity or gender. Furthermore, the effects of varying degrees of severity of $\mathrm{AD}$ on the differences in hippocampal gene expression were not taken into account. However, the present integrated analysis of different datasets of hippocampal gene expression in patients with AD may have facilitated the detection of genes that would have been missed in the analysis of a single patient or study cohort. Despite these limitations, the present study provided novel information regarding the molecular mechanisms of AD; however, further analyses are required to confirm the present findings.

In conclusion, the present study performed an integrated analysis, which provided significant insight into the global molecular changes associated with AD pathology. Furthermore, the present study identified DEGs as well as other biological functions, which may contribute to the successful identification of diagnostic factors or therapeutic targets for AD and the development of effective targeted therapies. Further functional 
studies may provide additional insight into the role of the DEGs in the pathophysiology of AD.

\section{References}

1. Hommet C, Mondon K, Constans T, Beaufils E, Desmidt T, Camus V and Cottier JP: Review of cerebral microangiopathy and Alzheimer's disease: Relation between white matter hyperintensities and microbleeds. Dement Geriatr Cogn Disord 32: 367-378, 2011

2. Hyman BT, Van Hoesen GW, Damasio AR and Barnes CL: Alzheimer's disease: Cell-specific pathology isolates the hippocampal formation. Science 225: 1168-1170, 1984.

3. Kordower JH, Chu Y Stebbins GT, DeKosky ST, Cochran EJ, Bennett D and Mufson EJ: Loss and atrophy of layer II entorhinal cortex neurons in elderly people with mild cognitive impairment Ann Neurol 49: 202-213, 2001

4. Scheff SW, Price DA, Schmitt FA, DeKosky ST and Mufson EJ: Synaptic alterations in CA1 in mild Alzheimer disease and mild cognitive impairment. Neurology 68: 1501-1508, 2007.

5. Kerchner GA, Hess CP, Hammond-Rosenbluth KE, Xu D, Rabinovici GD, Kelley DA, Vigneron DB, Nelson SJ and Miller BL: Hippocampal CA1 apical neuropil atrophy in mild Alzheimer disease visualized with 7-T MRI. Neurology 75: 1381-1387, 2010.

6. O'Brien RJ and Wong PC: Amyloid precursor protein processing and Alzheimer's disease. Annu Rev Neurosci 34: 185-204, 2011.

7. Noble W, Hanger DP, Miller CC and Lovestone S: The importance of tau phosphorylation for neurodegenerative diseases. Front Neurol 4: 83, 2013.

8. Martin L, Latypova X, Wilson CM, Magnaudeix A, Perrin ML, Yardin $\mathrm{C}$ and Terro $\mathrm{F}$ : Tau protein kinases: Involvement in Alzheimer's disease. Ageing Res Rev 12: 289-309, 2013.

9. Liu CC, Kanekiyo T, Xu H and Bu G: Apolipoprotein E and Alzheimer disease: Risk, mechanisms and therapy. Nat Rey Neurol 9: 106-118, 2013.

10. Auld DS, Kornecook TJ, Bastianetto S and Quirion R: Alzheimer's disease and the basal forebrain cholinergic system: Relations to beta-amyloid peptides, cognition and treatment strategies. Prog Neurobiol 68: 209-245, 2002.

11. Beckmann L, Fischer C, Deck KG, Nolte IM, te Meerman G and Chang-Claude J: Exploring haplotype sharing methods in general and isolated populations to detect gene(s) of a complex genetic trait. Genet Epidemiol 21 (Suppl 1): S554-S559, 2001

12. Blalock EM, Geddes JW, Chen KC, Porter NM, Markesbery WR and Landfield PW: Incipient Alzheimer's disease: Microarray correlation analyses reveal major transcriptional and tumor suppressor responses. Proc Natl Acad Sci USA 101: 2173-2178, 2004

13. Colangelo V, Schurr J, Ball MJ, Pelaez RP, Bazan NG and Lukiw WJ: Gene expression profiling of 12633 genes in Alzheimer hippocampal CA1: Transcription and neurotrophic factor down-regulation and up-regulation of apoptotic and pro-inflammatory signaling. J Neurosci Res 70: 462-473, 2002.

14. Mufson EJ, Counts SE and Ginsberg SD: Gene expression profiles of cholinergic nucleus basalis neurons in Alzheimer's disease. Neurochem Res 27: 1035-1048, 2002.

15. Siddiqui AS, Delaney AD, Schnerch A, Griffith OL, Jones SJ and Marra MA: Sequence biases in large scale gene expression profiling data. Nucleic Acids Res 34: e83, 2006.

16. Barrett T, Wilhite SE, Ledoux P, Evangelista C, Kim IF, Tomashevsky M, Marshall KA, Phillippy KH, Sherman PM, Holko M, et al: NCBI GEO: Archive for functional genomics data sets-update. Nucleic Acids Res 41: D991-D995, 2013.

17. Altermann E and Klaenhammer TR: PathwayVoyager: Pathway mapping using the Kyoto Encyclopedia of Genes and Genomes (KEGG) database. BMC Genomics 6: 60, 2005.
18. Tabas-Madrid D, Nogales-Cadenas R and Pascual-Montano A GeneCodis3: A non-redundant and modular enrichment analysis tool for functional genomics. Nucleic Acids Res 40: W478-W483, 2012.

19. Giot L, Bader JS Brouwer C, Chaudhuri A, Kuang B, Li Y, Hao YL, Ooi CE, Godwin B, Vitols E, et al: A protein interaction map of Drosophila melanogaster. Science 302: 1727-1736, 2003.

20. Schaefer MH, Lopes TJ, Mah N, Shoemaker JE, Matsuoka Y, Fontaine JF, Louis-Jeune C, Eisfeld AJ, Neumann G, Perez-Iratxeta $\mathrm{C}$, et al: Adding protein context to the human protein-protein interaction network to reveal meaningful interactions. PLoS Comput Biol 9: e1002860, 2013.

21. Shannon P, Markiel A, Ozier O, Baliga NS, Wang JT, Ramage D, Amin N, Schwikowski B and Ideker T: Cytoscape: A software environment for integrated models of biomolecular interaction networks. Genome Res 13: 2498-2504, 2003.

22. Miller JA, Woltjer RL, Goodenbour JM, Horvath S and Geschwind DH: Genes and pathways underlying regional and cell type changes in Alzheimer's disease. Genome Med 5: 48, 2013.

23. Hokama M, Oka S, Leon J, Ninomiya T, Honda H, Sasaki K, Iwaki T, Ohara T, Sasaki T, LaFerla FM, et al: Altered expression of diabetes-related genes in Alzheimer's disease brains: The Hisayama study. Cereb Cortex 24: 2476-2488, 2014.

24. Liang WS, Dunckley T, Beach TG, Grover A, Mastroeni D, Walker DG, Caselli RJ, Kukull WA, McKeel D, Morris JC, et al: Gene expression profiles in anatomically and functionally distinct regions of the normal aged human brain. Physiol Genomics 28: 311-322, 2007.

25. Meagher MJ, Schumacher JM, Lee K, Holdcraft RW, Edelhoff S, Disteche $\mathrm{C}$ and Braun RE: Identification of ZFR, an ancient and highly conserved murine chromosome-associated zinc finger protein. Gene 228: 197-211, 1999.

26. Kleines M, Gärtner A, Ritter K and Schaade L: Cloning and expression of the human single copy homologue of the mouse zinc finger protein zfr. Gene 275: 157-162, 2001.

27. Novarino G, Fenstermaker AG, Zaki MS, Hofree M, Silhavy JL, Heiberg AD, Abdellateef M, Rosti B, Scott E, Mansour L, et al: Exome sequencing links corticospinal motor neuron disease to common neurodegenerative disorders. Science 343: 506-511, 2014.

28. Zimmer DB, Chaplin J, Baldwin A and Rast M: S100-mediated signal transduction in the nervous system and neurological diseases. Cell Mol Biol (Noisy-le-grand) 51: 201-214, 2005.

29. Boom A, Pochet R, Authelet M, Pradier L, Borghgraef P, Van Leuven F, Heizmann CW and Brion JP: Astrocytic calcium/zinc binding protein S100A6 over expression in Alzheimer's disease and in PS1/APP transgenic mice models. Biochim Biophys Acta 1742: 161-168, 2004.

30. Bellucci C, Lilli C, Baroni T, Parnetti L, Sorbi S, Emiliani C, Lumare E, Calabresi P, Balloni S and Bodo M: Differences in extracellular matrix production and basic fibroblast growth factor response in skin fibroblasts from sporadic and familial Alzheimer's disease. Mol Med 13: 542-550, 2007.

31. Leduc V, Legault V, Dea D and Poirier J: Normalization of gene expression using SYBR green qPCR: A case for paraoxonase 1 and 2 in Alzheimer's disease brains. J Neurosci Methods 200: $14-19,2011$

32. Bamburg JR and Bloom GS: Cytoskeletal pathologies of Alzheimer disease. Cell Motil Cytoskeleton 66: 635-649, 2009.

33. Liang WS, Reiman EM, Valla J, Dunckley T, Beach TG, Grover A, Niedzielko TL, Schneider LE, Mastroeni D, Caselli R, et al: Alzheimer's disease is associated with reduced expression of energy metabolism genes in posterior cingulate neurons. Proc Natl Acad Sci USA 105: 4441-4446, 2008. 